\title{
A User Centered Innovation Approach Identifying Key User Values for the E-Newspaper
}

\author{
Carina Ihlström Eriksson, Halmstad University, Sweden \\ Jesper Svensson, Halmstad University, Sweden
}

\begin{abstract}
We have studied the pre-adoption phase of the e-newspaper, i.e. a newspaper published with e-paper technology. The research question of this article is: In what way can a user centered innovation process contribute to identifying key values in mobile innovations? The aim of this article is threefold: firstly, to identify key values for the e-newspaper, secondly, to examine the intention to adopt a new possible innovation and thirdly, to explore user centered design processes ability to capture user values early in the innovation process. We have identified seven key user values: mobility, availability, personalization, usability, interactivity, readability and environment. Furthermore, we found that surprisingly many would consider exchanging their traditional newspaper with an e-newspaper in the future, indicating that the e-newspaper have a fair chance of becoming an important innovation. Finally, we propose a four phase model for identifying user values in a pre-adoption phase of an innovation. [Article copies are available for purchase from InfoSci-on-Demand.com]
\end{abstract}

Keywords: E-Newspaper; Innovation; Pre-Adoption; User Centered; User Values

\section{INTRODUCTION}

During the last decade we have experienced how the internet and mobile technologies have changed the way we live, work and communicate. This evolution has made it possible for us to have anytime, anywhere access to information and applications.
There are three broad trends that are driving economy when it comes to mobile applications: changing customer priorities, new hardware/device innovation, and new infrastructure innovation (Kalakota \& Robinson, 2001). The ongoing diffusion of personal computers, handheld devices, and mobile telephones (Lyytinen \& Yoo, 2002) 
as well as the advent of new technologies such as the e-paper, enables new innovation possibilities in the mobile business (m-business) area.

However, despite all the innovation possibilities the adoption of mobile services/applications has not taken off as expected in Europe (Hammond, 2001; Carlsson et al., 2006). A growing body of research into adoption of mobile services has sought to understand the reasons for this slow adoption rate (Sarker \& Wells, 2003; Vrechopoulos et al., 2003; Carlsson et al., 2005; Mallat, 2006; Blechar et al., 2006). Among the explanations as to why this adoption rate is low, usability factors, technological factors and business model factors are mentioned. As use patterns changes, new demands and expectations emerge, which lead to uncertainty about what people actually value and are willing to pay for (Tilson et al., 2004)

To guarantee a successful m-business application the designers/managers need to have a complete understanding of the mobile business value including the experience of the user (Sims et al., 2007). Kalakota and Robinson (2001) suggest several fundamental questions in elucidating customer experience, e.g. the relationship between customer experience and perceived value; what features is the customer looking for; and what is the customer willing to pay for the experience. The value-in use concept focuses on the experience perceived by a user interacting with products or services in use situations. According to this concept, the customers experience and perception are essential to be able to determinate user value (Kuusisto, 2008). Consumer value can also be defined in terms of the monetary sacrifice people are willing to make for a product (Boztepe, 2007). Indeed, identifying key user values early and emphasize these values in the innovation process could make the difference between a success and a failure.

Opening up innovation processes to involve users and other relevant stakeholders as innovators are recognized to be important to meet the user needs and leverage market value (von Hippel, 2005; Gassman, 2006; Lettl et al., 2006). End users have specific domain knowledge that in many cases other stakeholders lack. Therefore, this group is in many cases an essential source for innovation. Involving users in the innovation process could increase the possibilities of identifying key values of products and services, which in turn enhance the odds to make it into the market and to be adopted by the users.

One example of an industry seeking new innovations is the newspaper industry. Lately, this industry has faced declining subscription and advertising revenues, forcing it to seek new alternative ways to profit and to attract new audiences (WAN, 2006; The Economist, 2006). The advent of e-paper technology has captured the interest of the industry (IFRA eNews initiative, 2006; The Swedish Newspaper Publishers' Association, 2007). The main advantage with e-paper technology is that it is reflecting, giving the same reader experience as paper and is thin, flexible and non-sensitive with WiFi connection, making it a device suitable for $\mathrm{m}$-business applications. The potential replacement of the traditional newspaper with the e-newspaper, i.e. a newspaper published with e-paper technology, in the future would dramatically reduce production and distribution costs, making it an interesting prospect. Moreover, there is a belief within the industry that the e-newspaper could reach new audiences, e.g. young people. 
39 more pages are available in the full version of this document, which may be purchased using the "Add to Cart" button on the publisher's webpage:

www.igi-global.com/article/user-centered-innovationapproach-identifying/3941

\section{Related Content}

Culture and Consumer Trust in Online Businesses

Robert Greenberg, Bernard Wong-On-Wing and Gladie Lui (2010). Electronic Services: Concepts, Methodologies, Tools and Applications (pp. 1402-1421). www.irma-international.org/chapter/culture-consumer-trust-onlinebusinesses/44023/

Economy Based Resource Allocation in laaS Cloud Hemant Kumar Mehta and Eshan Gupta (2013). International Journal of Cloud Applications and Computing (pp. 1-11).

www.irma-international.org/article/economy-based-resource-allocation-iniaas-cloud/81237/

Impact of Wireless Sensor Network Technology on Service Innovation in Supply Chain Management

Gong Li and Jing Shi (2010). Service Science and Logistics Informatics: Innovative Perspectives (pp. 65-96).

www.irma-international.org/chapter/impact-wireless-sensor-networktechnology/42636/

Media Richness in Online Consumer Interactions: An Exploratory Study of Consumer-Opinion Web Sites

Irene Pollach (2010). Electronic Services: Concepts, Methodologies, Tools and Applications (pp. 1472-1489).

www.irma-international.org/chapter/media-richness-online-consumerinteractions/44026/

Servitized Enterprises for Distributed Collaborative Commerce Haluk Demirkan and James C. Spohrer (2010). International Journal of Service Science, Management, Engineering, and Technology (pp. 68-81).

www.irma-international.org/article/servitized-enterprises-distributedcollaborative-commerce/41009/ 